OPEN ACCESS

Edited by:

Guang Yang,

Imperial College London,

United Kingdom

Reviewed by:

Yi Zhang,

Sichuan University, China

Shouping Zhu,

Xidian University, China

T. Niu,

Georgia Institute of Technology,

United States

*Correspondence:

Zhanli Hu

zl.hu@siat.ac.cn

${ }^{\dagger}$ These authors have contributed equally to this work

Specialty section:

This article was submitted to

Cancer Imaging and Image-directed Interventions,

a section of the journal

Frontiers in Oncology

Received: 19 November 2021 Accepted: 27 December 2021

Published: 26 January 2022

Citation:

Deng $F$, Li X, Yang $F$, Sun $H$, Yuan J, He Q, Xu W, Yang Y, Liang D, Liu X, Mok GSP, Zheng $H$ and $H u Z$ (2022)

Low-Dose 68 Ga-PSMA Prostate PET/MRI Imaging Using Deep Learning Based on MRI Priors.

Front. Oncol. 11:818329. doi: 10.3389/fonc.2021.818329

\section{Low-Dose 68 Ga-PSMA Prostate PET/MRI Imaging Using Deep Learning Based on MRI Priors}

Fuquan Deng ${ }^{1,2,3 \dagger}$, Xiaoyuan $\mathrm{Li}^{4 \dagger}$, Fengjiao Yang ${ }^{4}$, Hongwei Sun ${ }^{5}$, Jianmin Yuan ${ }^{6}$, Qiang $\mathrm{He}^{6}$, Weifeng $\mathrm{Xu}^{2}$, Yongfeng Yang ${ }^{1,3}$, Dong Liang ${ }^{1,3}$, Xin Liu ${ }^{1,3}$, Greta S. P. Mok ${ }^{7}$, Hairong Zheng ${ }^{1,3}$ and Zhanli $\mathrm{Hu}^{1,3 *}$

${ }^{1}$ Lauterbur Research Center for Biomedical Imaging, Shenzhen Institute of Advanced Technology, Chinese Academy of Sciences, Shenzhen, China, ${ }^{2}$ Computer Department, North China Electric Power University, Baoding, China, ${ }^{3}$ Chinese Academy of Sciences Key Laboratory of Health Informatics, Shenzhen, China, ${ }^{4}$ Department of Nuclear Medicine, Nanjing First Hospital, Nanjing Medical University, Nanjing, China, 5 United Imaging Research Institute of Intelligent Imaging, Beijing, China, ${ }^{6}$ Central Research Institute, United Imaging Healthcare Group, Shanghai, China, ${ }^{7}$ Biomedical Imaging Laboratory (BIG), Department of Electrical and Computer Engineering, Faculty of Science and Technology, University of Macau, Avenida da Universidade, Macau SAR, China

Background: 68 Ga-prostate-specific membrane antigen (PSMA) PET/MRI has become an effective imaging method for prostate cancer. The purpose of this study was to use deep learning methods to perform low-dose image restoration on PSMA PET/MRI and to evaluate the effect of synthesis on the images and the medical diagnosis of patients at risk of prostate cancer.

Methods: We reviewed the 68 Ga-PSMA PET/MRI data of 41 patients. The low-dose PET (LDPET) images of these patients were restored to full-dose PET (FDPET) images through a deep learning method based on MRI priors. The synthesized images were evaluated according to quantitative scores from nuclear medicine doctors and multiple imaging indicators, such as peak-signal noise ratio (PSNR), structural similarity (SSIM), normalization mean square error (NMSE), and relative contrast-to-noise ratio (RCNR).

Results: The clinical quantitative scores of the FDPET images synthesized from 25\%-and $50 \%$-dose images based on MRI priors were $3.84 \pm 0.36$ and $4.03 \pm 0.17$, respectively, which were higher than the scores of the target images. Correspondingly, the PSNR, SSIM, NMSE, and RCNR values of the FDPET images synthesized from 50\%-dose PET images based on MRI priors were 39.88 $\pm 3.83,0.896 \pm 0.092,0.012 \pm 0.007$, and $0.996 \pm 0.080$, respectively.

Conclusion: According to a combination of quantitative scores from nuclear medicine doctors and evaluations with multiple image indicators, the synthesis of FDPET images based on MRI priors using and 50\%-dose PET images did not affect the clinical diagnosis of prostate cancer. Prostate cancer patients can undergo 68 Ga-PSMA prostate PET/MRI scans with radiation doses reduced by up to $50 \%$ through the use of deep learning methods to synthesize FDPET images.

Keywords: PET/MRI, prostate, low-dose restoration, deep learning, discrete wavelet transform 


\section{INTRODUCTION}

Prostate cancer is one of the most common cancers worldwide, with approximately 1.41 million new cases reported in 2020, the third most common among 36 cancers (1). In recent years, studies have demonstrated that $68 \mathrm{Ga}$-prostate-specific membrane antigen (PSMA) PET/MRI provides accurate staging of primary prostate cancer with a high detection rate (2-6). In terms of evaluating recurrent prostate cancer, this imaging technique also has a high detection rate, even for patients with extremely low levels of prostate specific antigen (PSA; $<0.5 \mathrm{ng} / \mathrm{ml}$ ). Additionally, it plays an important role in tumor detection, preliminary staging, treatment response assessment, and treatment planning $(7,8)$.

However, this technique also has some limitations, including scanning time, the cost of the associated radiopharmaceuticals, and the radiation delivered by PET imaging (9). The economic factors and radiation risks have different kinds of impact on the patients. The purpose of reducing the dose of radiopharmaceuticals is related to the potential risks of ionizing radiation. To reduce the risk of radiation exposure that those involved in the scan may face, especially pediatric patients and volunteers, or when a variety of different tracers are used for follow-up or to monitor treatment response, fewer radiopharmaceuticals should be used to perform PET imaging. The reduction in the number of radiopharmaceuticals will reduce the quality of the PET images, thereby affecting quantitative analysis and clinical diagnosis.

In recent years, deep learning has entered various fields of medical imaging. Hu Chen et al. used learned experts' assessment-based reconstruction network to reconstruct CT directly from sinogram data (10). Maosong Ran et al. proposed a Parallel Dual-Domain Convolutional Neural Network for Compressed Sensing MRI to deal with the k-space and spatial data simultaneously (11) and a parameter-dependent framework to process the CT data with different scanning geometries and dose level in a unified network (12). Wenjun Xia et al. simultaneously leverage the spatial convolution to extract the local pixel-level features from the images and incorporate the graph convolution to analyze the nonlocal topological features in manifold space for low-dose CT reconstruction (13). Chenyu Shen et al. leveraged deep regularization by denoising from a Bayesian perspective to reconstruct PET images from a single corrupted sinogram without any supervised or auxiliary information (14). Researchers have proposed a variety of methods to ensure that the synthesized FDPET images have the same image quality as the clinical diagnostic images (15-17). In particular, deep learning has shown great potential in recovering LDPET images. Yan Wang et al. used a $3 \mathrm{D}$ conditional generative adversarial network (GAN) to synthesize FDPET images from head LDPET images (18). Wenzhuo $\mathrm{Lu}$ et al. used a fully optimized $3 \mathrm{D} \mathrm{U}$-net to effectively reduce the noise in LDPET images from the lungs while minimizing the deviation in the lung nodules (19). Yang Lei et al. proposed using CycleGAN to estimate prostate FDPET images from prostate LDPET images (20). Long Zhou et al. also used CycleGAN, denoising low-dose fluorodeoxyglucose (FDG)
PET images and subsequently performing quantitative analysis on the images (21).

Deep learning also has a variety of exploratory research in prostate PET images. Pablo Borrelli et al. used convolutional neural network to detect lymph node metastases by PET/CT predicting prostate cancer-specific survival (22). Eirini Polymeri et al. evaluated a novel three-dimensional deep learning-based technique on PET/CT images for automated assessment of cancer in the prostate gland and its agreement with manual assessment (23). Sangwon Han et al. evaluated the performance of deep learning classifers for bone scans of prostate cancer patients (24). Dejan Kostyszyn et al. examined the capabilities of convolutional neural network for intraprostatic GTV contouring in 68Ga- and 18F-PSMA-PET (25). Sobhan Moazem et al. used UNet to predict treatment response in prostate cancer patients based on multimodal PET/CT for clinical decision support (26). Andrii Pozaruk et al. developed a novel augmented deep learning method based on GANs for accurate attenuation correction in the simultaneous PET/MR scanner (27).

In this study, we retrospectively analyzed the 68 Ga-PSMA PET/MRI data of 41 patients in Nanjing First Hospital of China. The PET images were reconstructed at acquisition times of $2.5 \%$, $5 \%, 25 \%, 50 \%$, and $100 \%$ of the standard acquisition time. A discrete-wavelet-transform convolutional neural network (DWTN) was used to restore the LDPET images to the original, FDPET images with or without the use of MRI priors, respectively, to explore the extent to which this method can reduce the required radiotracer dose.

\section{MATERIALS AND METHODS}

\section{PET/MRI Data Acquisition}

In this study, we used clinical images obtained from $68 \mathrm{Ga}$ PSMA PET/MRI examinations performed at Nanjing First Hospital of China from January 2021 to July 2021. The data were obtained from 41 male patients who might present with signs of future prostate cancer. The mean age of the patients was $67 \pm 6$ years, and the mean weight was $73 \pm 10 \mathrm{~kg}$. The research protocol was approved by the institutional ethics committee, and all patients were provided written informed content. Sixty minutes after the patient had been injected with 68 Ga-PSMA (in the range of $111-185 \times 10^{6} \mathrm{MBq}$ ), scanning data were collected from the PET/MRI scanner (United Imaging Healthcare, uPMR 790). The acquisition time of emission images was 600 seconds, and the PET images were reconstructed at acquisition times of $600,300,150,30$, and 15 seconds.

All PET images were reconstructed using ordered subset expectation maximization (OSEM) algorithm and a set series of parameters, for example, 3D iterative time-of-flight (TOF) and point-spread function (PSF) reconstruction, 2 iterations, 20 subsets, matrix $192 \times 192$, slice thickness $2.5 \mathrm{~mm}$, and correction methods such as decay correction, attenuation correction, scatter correction, dead time correction, random correction, and detector normalization correction. The images reconstructed at the acquisition times above correspond to 100\%-, 50\%-, 25\%-, 5\%-, 
and 2.5\%-dose (low-dose) PET images, respectively. The 100\% does PET images were used as ground-truth and the remaining dose PET images were used as input images. The water sequence decomposed from the T1-weighted MRI images was used as the prior images for generating the LDPET images.

Since integrated prostate PET/MRI was used for scanning, the PET and MRI scans were coaxial. The PET image matrix size is $192 \times 192$, and the MRI image matrix size is $552 \times 387$. To ensure that the image resolution was not lower than that of the original image, we used bicubic interpolation to resize the two modal images as a $512 \times 512$ matrix. Since the image matrix sizes of the two modalities are now the same, the images of the two modalities do not need to be registered. In total, the 41 patients had 4100 sets of images. These sets are divided into training data set and test data set. We used $90 \%$ of the data set to train the model and the remaining $10 \%$ to verify the effect of the images generated by the model. To avoid overfitting due to the small size of the training data set, we increased the number of images in the training data set by flipping the images down, left and right, quadrupling the size of the training data set. This process helped improve the generalizability of the deep learning models.

\section{Discrete-Wavelet-Transform Convolutional Neural Network}

The discrete-wavelet-transform convolutional neural network (DWTN) proposed in this study is an improvement of the densely self-guided wavelet network (28), which is suitable for LDPET image restoration tasks based on MRI priors. The structure of DWTN was shown in Figure 1. The multilayer selfguided architecture makes better use of multiscale image information; low-resolution feature information from the top layer is gradually fused with higher-resolution feature information to improve the network's ability to extract multiscale feature information from images. Wavelet transform is used instead of ordinary upsampling and downsampling and PixelShuffle and PixelUnshuffle to generate multiscale image information. Before the convolution process, the image is

A

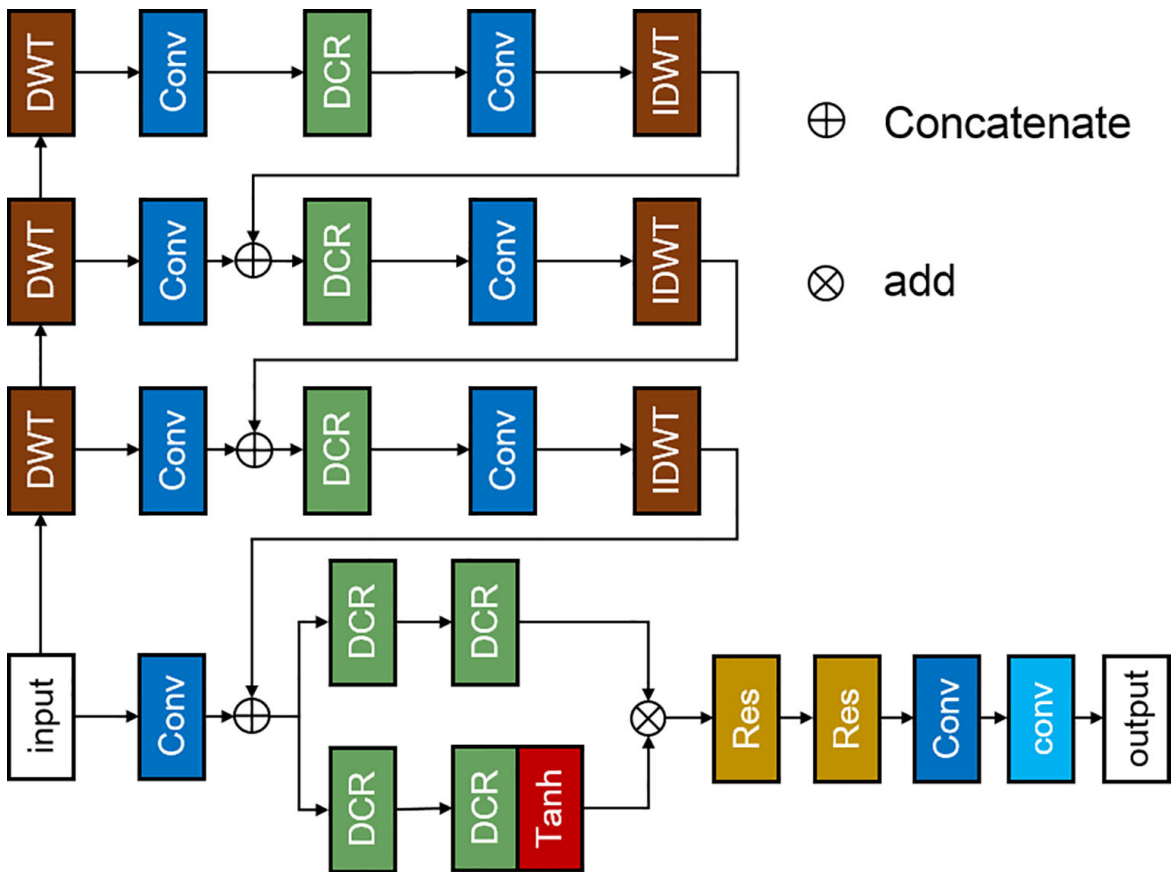

B

C

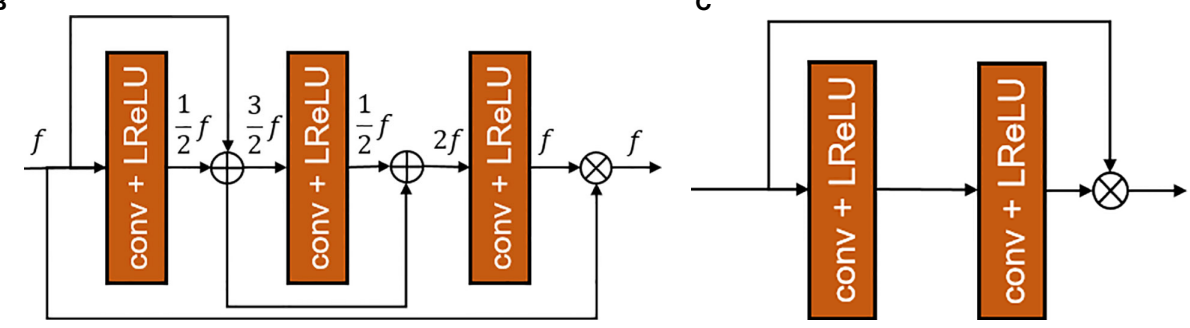

FIGURE 1 | Discrete-wavelet-transform neural network (DWTN), including (A) the structure of the DWTN network, (B) the structure of the densely connected residual (DCR) block, and $\mathbf{( C )}$ the structure of the residual block. 
converted into horizontal, vertical, and diagonal detail images and thumbnails through discrete wavelet transform (29). At the fullresolution layer, the main branch and attention branch provide stability and process the feature images. At each layer, we add densely connected residual blocks to improve the convergence of the network. The top layer of the DWTN extracts large-scale image feature information in the lowest resolution space. The toplevel network contains two convolution layers, a leaky ReLU layer, and a densely connected residual (DCR) block (30). As shown in Figure 1 (B), the DCR block consists of three convolutional layers, each of which is followed by a leaky ReLU. Each feature image is connected by dense connections so that our model can use the previous feature information to solve the gradient disappearance problem. The middle two layers are similar to the top layer. For the full-resolution level, we add multiple DCR blocks after merging the multiscale feature information to enhance the feature extraction capability of the DWTN. For the attention branch, we add a tanh activation function after the two DCR blocks. The main branch and the attention branch are processed and added, and then through multiple residual blocks and convolution blocks, the added image feature information is extracted. The structure of residual block was shown in Figure 1 (C). Finally, through a convolution block without an activation function, the details of the image are preserved. The last convolution layer uses a $1 \times 1$ convolution kernel with a step count of 1 , and the remaining convolution layers all use a $3 \times 3$ convolution kernel with a step count of 1 .

To improve the generalization of the network, we used perceptual loss, mean-square-error loss and kernel loss to constrain the network (31). Among them, perceptual loss enabled the network to learn the characteristics of the overall images and converge faster than mean-absolute-error loss. The perceptual loss used pre-trained VGG19 for extracting image features. The mean-square-error loss was to calculate the loss function at the pixel level to ensure the network to generate more details of the images. The kernel loss reduced the weight of the hidden layer inside network, thereby promoting the convergence of DWTN. The loss function formula was as follows:

$$
\text { Loss }_{\text {total }}=m s e \times \operatorname{Loss}_{M S E}+v g g \times \text { Loss }_{\text {per }}+k l \times \text { Loss }_{k e r},
$$

where mse, vgg and $\mathrm{kl}$ are $0.5,0.5$ and 0.0001 , respectively. The formulas of each loss function are as follows:

$$
\begin{gathered}
\operatorname{Loss}_{M S E}=\mathbb{E}(\widehat{y}-y)^{2}, \\
\text { Loss }_{p e r}=\mathbb{E}(\operatorname{VGG}(\widehat{y})-\operatorname{VGG}(\mathrm{y}))^{2}, \\
\operatorname{Loss}_{k e r}=\mathbb{E}(\widehat{y})^{2},
\end{gathered}
$$

where $\hat{y}$ and $y$ represent the generated images and ground-truth, respectively. VGG represents the processing of VGG model.

When initializing the training parameters, we used the ADAM optimizer, and the remaining parameters are set to their default values. We update the learning rate every 25 epochs and set the learning rate decay rate to 0.5 . The weights of all hidden layers are initialized with Gaussian random numbers. The model was implemented on an NVIDIA GeForce RTX 2080Ti GPU with 11 GB of memory and run under the Microsoft Windows 10 operating system. During training, we used a batch size of 4 for 100 epochs.

\section{Evaluation Metrics}

Clinical quantitative evaluation. To evaluate the quality of the PET images, we evaluated the original LDPET images, the synthesized FDPET images, and the synthesized FDPET images based on the MRI priors. There were 13 sets of images, including 32 PET images in each group. The PET images were evaluated using a 5-point method by two nuclear medicine physicians from Nanjing First Hospital of China (32, 33).

Image quantitative analysis. To evaluate the image quality between the synthesized FDPET images and the original FDPET images, we used the PSNR, SSIM, RCNR, and NMSE as objective indicators.

The PSNR is a quantitative index for evaluating images and noise, and the SSIM is an index for evaluating the similarity of two image features; both indices offer comprehensive evaluations of two images.

$$
\text { PSNR }=10 \times \log _{10}\left(\frac{\left(2^{n}-1\right)^{2}}{M S E}\right),
$$

Where MSE is the mean square error between the compared images and the ground-truth.

$$
\operatorname{SSIM}=\frac{\left(2 \mu_{x} \mu_{y}+c_{1}\right)\left(2 \sigma_{x y}+c_{2}\right)}{\left(\mu_{x}^{2}+\mu_{y}^{2}+c_{1}\right)\left(\sigma_{x}^{2}+\sigma_{y}^{2}+c_{2}\right)}
$$

where $\mu_{x}$ is the mean of compared images and $\mu_{y}$ is the mean of ground-truth, respectively. $\sigma_{x}$ and $\sigma_{y}$ are variance of compared images and ground-truth, respectively. $\sigma_{x y}$ is the covariance between compared images and ground-truth. $c_{1}$ and $c_{2}$ are 0.01 and 0.03 , respectively.

The NMSE is an indicator of the quantitative analysis of two images at the pixel level.

$$
\mathrm{NMSE}=\frac{\sum_{i \in V}\left(X_{i}-Y_{i}\right)^{2}}{\sum_{i \in V}\left(Y_{i}\right)^{2}},
$$

Where $X_{i}$ and $Y_{i}$ represent the pixel value of the compared image and groud-truth, respectively.

The contrast-to-noise ratio is an objective index used to evaluate the quality of medical images; the RCNR is a dimensionless image index based on CNR that is used to compare the contrast of two images.

$$
\begin{gathered}
\mathrm{CNR}=\frac{X-X_{\text {background }}}{X} \\
\mathrm{RCNR}=\frac{C N R_{x}}{C N R_{y}},
\end{gathered}
$$

Where $\mathrm{X}$ and $X_{\text {background }}$ represent the mean of the matrix and background matrix, respectively. 


\section{RESULTS}

Compared with the LDPET images, the FDPET images synthesized by the deep learning method demonstrated significantly improved image quality. PET images with a dose of less than 5\% showed irregular spots, and their contours, shapes, and contrast were different from those of the target images. In the images synthesized from those with a dose of less than 5\%, the spots were eliminated, and the shape features and contrast were relatively consistent with those of the target images. In PET images with a dose greater than $5 \%$, the contours, shapes, and contrast were similar to those the target images, but subtle differences could be observed. In the synthesized PET images, the shapes, contours, and contrast were consistent with those of the target images.

After training the model, we calculated the mean and standard deviation of the RCNR, PSNR, SSIM, NMSE among the original LDPET original image, the synthesized FDPET images, the priorsynthesized FDPET images, and the target images of all doses in the test set. Table 1 shows the mean and standard deviation values of the image indicators. To more intuitively visualize the differences in these image indicators, Figure $\mathbf{2}$ shows the histograms of the structural similarity and NMSE indicators. The processed LDPET images had significantly better image quality than the original LDPET images. The images based on MRI prior synthesis showed better image quality at the global-feature and pixel levels.

Figure 3 shows one patient's original 2.5\%-, 5\%-, 25\%-, and $50 \%$-dose PET images and their corresponding synthesized FDPET images and prior-synthesized FDPET images for the prostate. The average RCNR of the LDPET images and MRI prior-based synthesized LDPET images was close to 1. Moreover, the pelvic contour details of the prior-based synthesized images for doses of $25 \%$ and above are visible.

We transferred the synthesized images and MRI prior synthesized images for each LDPET image to DICOM format, subtracted each image from the original FDPET image matrix, and finally divided the difference matrix by the maximum value of the original image. The resulting image matrixes are shown in Figure 4. The error between the $25 \%$-dose synthesized images and the prior-synthesized images are within $25 \%$, and the error between the 50\%-dose synthesized images and the priorsynthesized images are within $10 \%$.

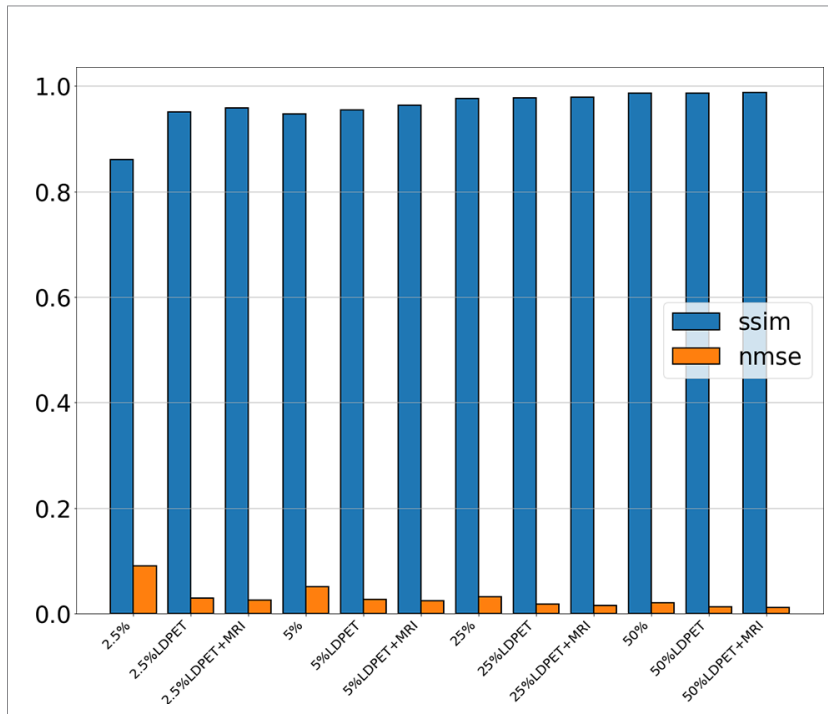

FIGURE 2 | NMSE and SSIM of the original LDPET images, synthetic FDPET images, and MR prior-synthesized FDPET images at all doses, where blue represents SSIM, orange represents NMSE, and different degrees of color represent images of different doses.

Six patients were selected from the test set, all of whom had images of prostate or pelvic lesions, as shown in Figure 5. The figure shows the diffusion weighted (DW) image, apparent diffusion coefficient (ADC) image, T2-weighted image, FDPET image, and various synthesized images. The DW image, ADC image, and T2-weighted image in the MRI sequence are important references for clinical diagnosis, and the PET images and MRI images are complementary.

In the clinical quantification phase, we selected 32 images with lesions in the pelvis or prostate from the test set for scoring. Table 2 shows the mean and variance of the scored from the two nuclear medicine doctors for PET images of different doses, processed PET images, and prior-based PET images. The average score of the FDPET images MRI prior-synthesized from 25\%-dose PET images is 0.1 points lower than the average score of the target images. In contrast, the average score of the FDPET images synthesized from $25 \%$ dose PET images without the prior is 0.3 points lower than the average score of the target images. In addition, to improve the

TABLE 1 | Objective indicators for LDPET images, synthesized FDPET images, MR prior-synthesized FDPET images, and target images.

\begin{tabular}{|c|c|c|c|c|}
\hline Image & PSNR & SSIM & NMSE & RCNR \\
\hline $2.5 \%$ & $25.56 \pm 4.99$ & $0.745 \pm 0.112$ & $0.065 \pm 0.045$ & $0.835 \pm 0.267$ \\
\hline 2.5\%LDPET & $32.51 \pm 4.89$ & $0.820 \pm 0.079$ & $0.029 \pm 0.021$ & $1.046 \pm 0.213$ \\
\hline 2.5\%LDPET+MRI & $33.34 \pm 4.47$ & $0.846 \pm 0.060$ & $0.026 \pm 0.018$ & $1.118 \pm 0.218$ \\
\hline $5 \%$ & $26.99 \pm 5.56$ & $0.756 \pm 0.113$ & $0.057 \pm 0.037$ & $0.860 \pm 0.227$ \\
\hline 5\%LDPET & $33.25 \pm 4.57$ & $0.814 \pm 0.137$ & $0.026 \pm 0.018$ & $1.032 \pm 0.216$ \\
\hline 5\%LDPET+MRI & $33.78 \pm 4.25$ & $0.817 \pm 0.141$ & $0.024 \pm 0.017$ & $0.964 \pm 0.248$ \\
\hline $25 \%$ & $29.58 \pm 7.05$ & $0.832 \pm 0.114$ & $0.047 \pm 0.036$ & $0.901 \pm 0.195$ \\
\hline 25\%LDPET & $36.90 \pm 4.40$ & $0.893 \pm 0.090$ & $0.017 \pm 0.012$ & $1.035 \pm 0.123$ \\
\hline 25\%LDPET+MRI & $37.86 \pm 4.16$ & $0.916 \pm 0.063$ & $0.015 \pm 0.012$ & $1.004 \pm 0.126$ \\
\hline $50 \%$ & $32.02 \pm 8.66$ & $0.865 \pm 0.123$ & $0.040 \pm 0.036$ & $0.909 \pm 0.183$ \\
\hline 50\%LDPET & $39.48 \pm 3.90$ & $0.919 \pm 0.067$ & $0.012 \pm 0.008$ & $1.009 \pm 0.079$ \\
\hline 50\%LDPET+MRI & $39.88 \pm 3.83$ & $0.896 \pm 0.092$ & $0.012 \pm 0.007$ & $0.996 \pm 0.080$ \\
\hline
\end{tabular}

In bold: The best performance in this indicator. 


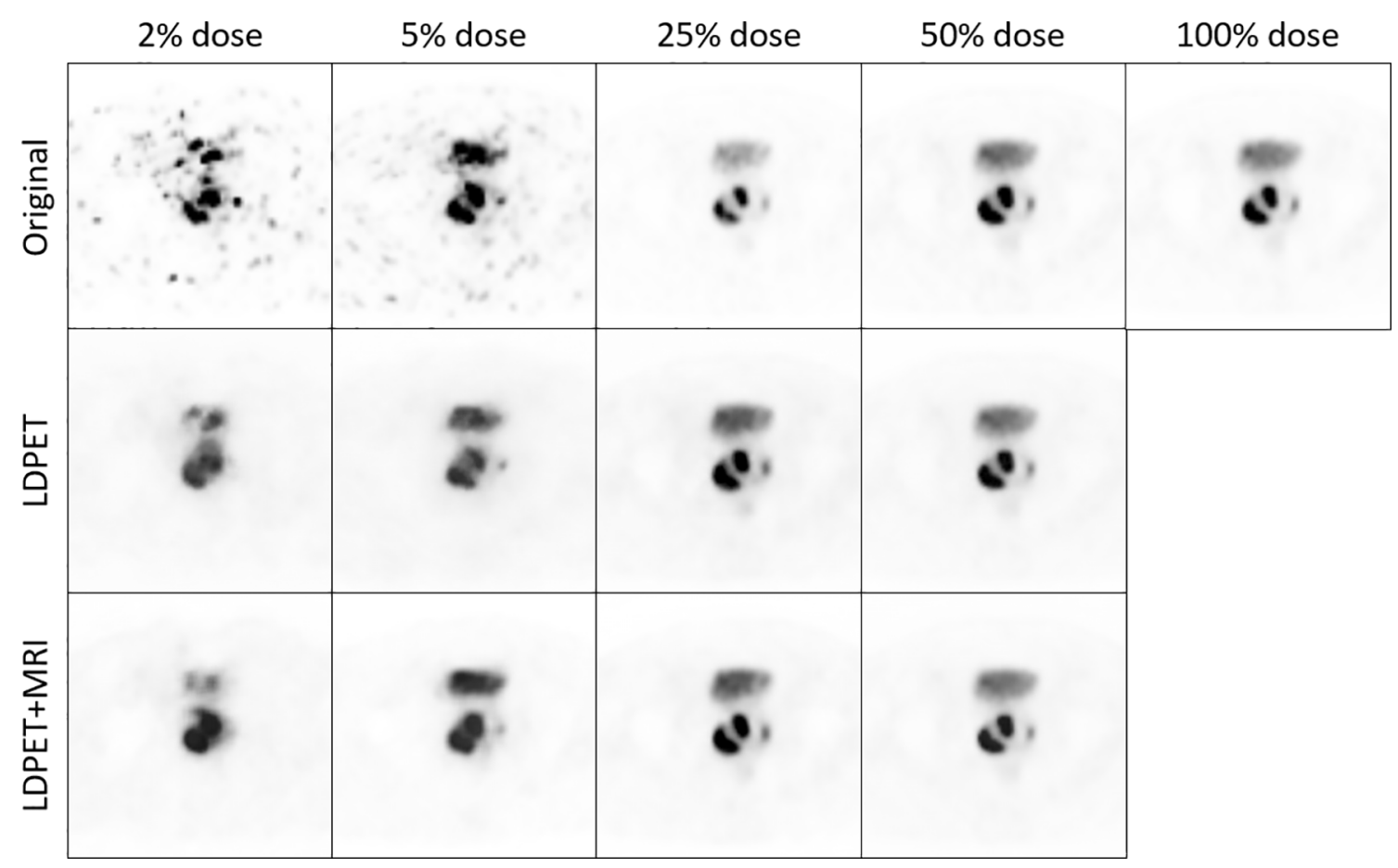

FIGURE 3 | The original LDPET images, synthesized FDPET images, MR prior-synthesized FDPET images of all doses, and their ROls.

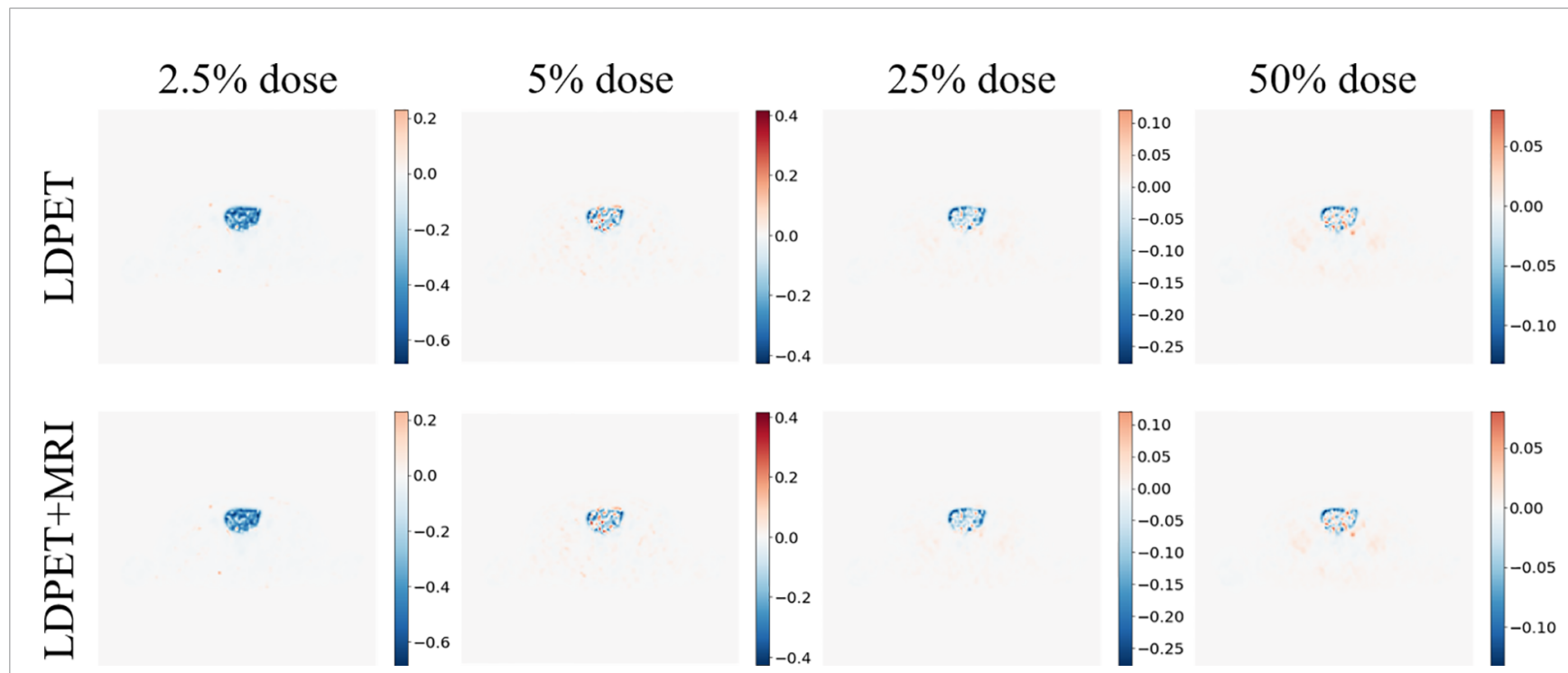

FIGURE 4 | Synthesized FDPET image, MR prior for all doses, combined FDPET image and target subtraction difference map and original image.

credibility of the analysis, we combined the scores of the two doctors. When the two nuclear medicine doctors had different scores for the same image, then the lower score of the two was taken. Figure 6 shows the distribution of this score. When MRI images were used as the prior, more than $80 \%$ of the FDPET images synthesized from $25 \%$-dose PET images had scores of 4 , while the rest had scores of 3 . When no priors were used, the scores of the FDPET images synthesized from the $25 \%$-dose images indicated that the FDPET images were between good and poor quality. Regardless of whether MRI images were used as priors, the scores of the FDPET image synthesized from the 50\%-dose PET images were greater than or equal to 4 . At each dose, using MRI images as 


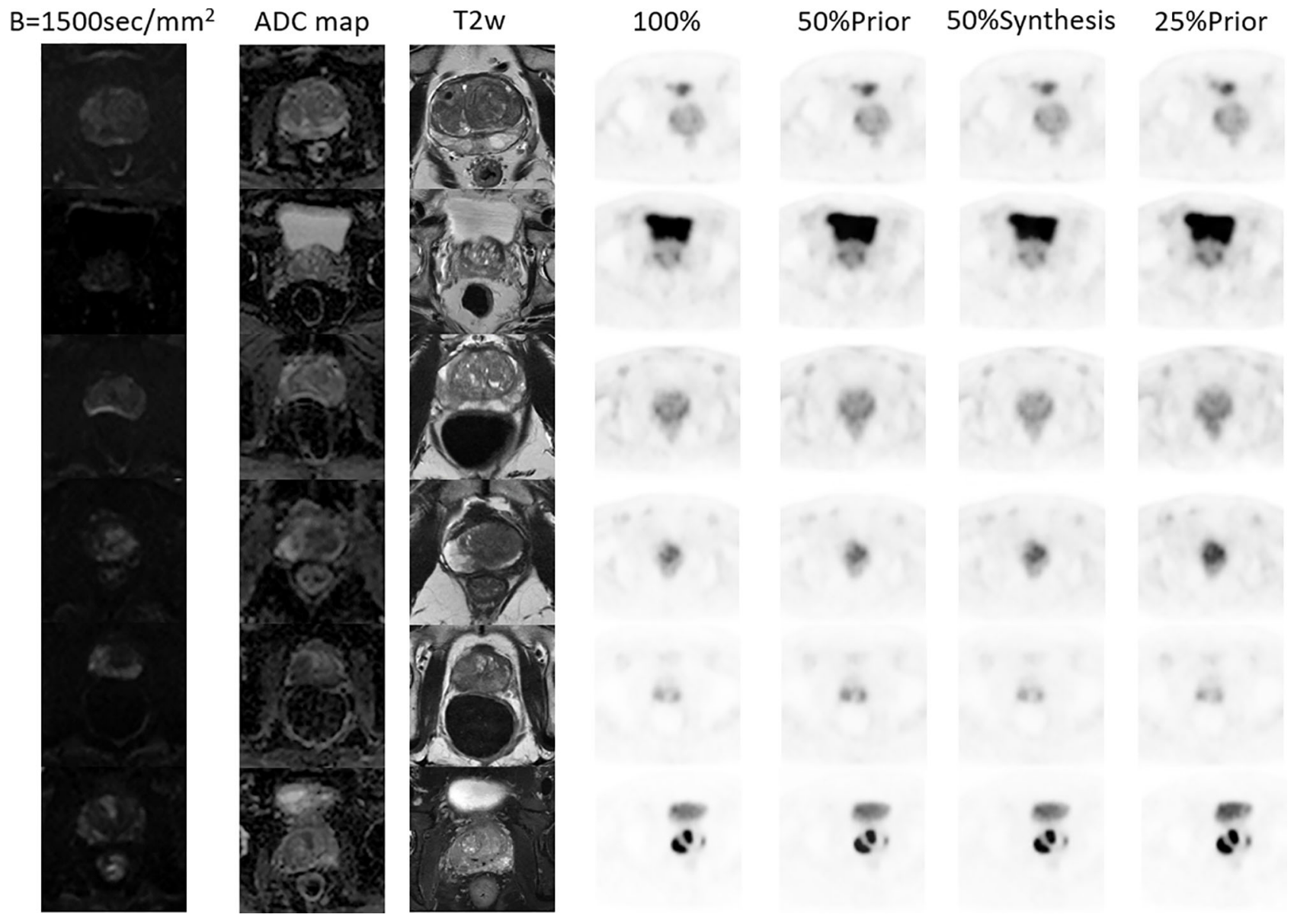

FIGURE 5 | MR and PET images of 6 patients with prostate or pelvic lesions from the test set. The MR sequences included DW-, ADC- and T2-weighted images. The PET images consist of the original FDPET images, FDPET images synthesized from $50 \%$-dose images with MR priors, FDPET images synthesized from $50 \%$ dose images without MR priors, and FDPET images synthesized from 25\%-dose images with MR priors.

TABLE 2 | Mean clinical quantitative scores from nuclear medicine doctors on LDPET images, synthesized FDPET images, MR prior-synthesized FDPET images, and target images.

\begin{tabular}{lccc}
\hline & Original & LDPET & LDPET+MRI \\
\hline $2.5 \%$ & $1.00 \pm 0.00$ & $2.44 \pm 0.56$ & $2.69 \pm 0.46$ \\
$5 \%$ & $1.69 \pm 0.46$ & $2.94 \pm 0.43$ & $3.28 \pm 0.57$ \\
$25 \%$ & $2.47 \pm 0.50$ & $3.62 \pm 0.48$ & $3.84 \pm 0.36$ \\
$50 \%$ & $3.03 \pm 0.39$ & $4.03 \pm 0.17$ & $4.03 \pm 0.17$ \\
$100 \%$ & $3.94 \pm 0.24$ & - & - \\
\hline
\end{tabular}

priors in synthesizing the images was better than using single LDPET images to synthesize FDPET images.

\section{DISCUSSION}

In the LDPET image denoising method, MRI images were used as prior image to provide rich tissue and anatomical information for PET image synthesis, thereby improving image quality, contours and details. However, the use of MRI as priors can also lead to registration problems. Due to the characteristics of the MRI images and the PSMA PET images, accurate registration, regardless of whether rigid registration or flexible registration is used, can be difficult. Data registration is not a problem, however, because the integrated PET/MRI device uses coaxial scanning, and the data it collects can be directly applied to LDPET restoration. Theoretically, data collected by integrated PET/MRI are more suitable for MRI prior-based LDPET estimation than data collected by sequential PET/MRI, insert PET/MRI, or multiple devices.

When using a single LDPET image for denoising, synthesized prostate FDPET images have poor overall contour and edge details and cannot be restored well. When including MRI images as priors for LDPET image denoising, the edges of PET images with a dose of less than $5 \%$ is blurred, and the surrounding contours are not clear, which can affect the diagnosis. In PET images with a dose higher than $5 \%$, the shape and edges of the key parts of the prostate are clear, the contours of the surrounding organs are distinct, the contrast is relatively close to that of the target image, and the clinical quantitative score is the same as that of the target 


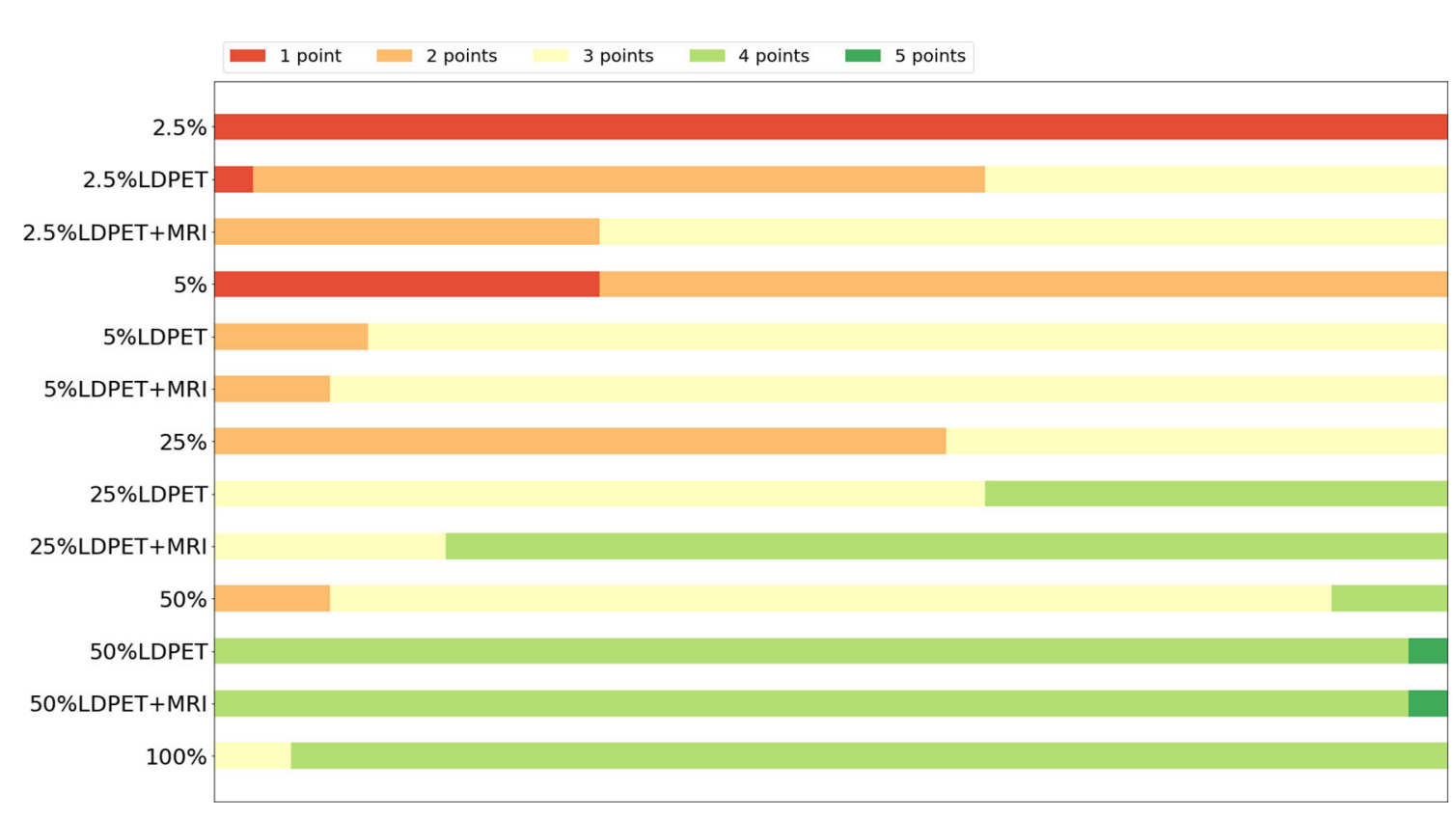

FIGURE 6 | Distribution of clinical quantitative scores from nuclear medicine doctors on LDPET images, synthesized FDPET images, MR prior-synthesized FDPET images, and target images.

image. However, Figure 3 shows that the 25\%-dose PET synthesized images based on the MRI priors and the groundtruth have a large error in the local area of the prostate. Thus, the $50 \%$-dose PET images synthesized based on the MRI priors showed sufficient quality to meet the requirements for clinical analysis.

However, our method still has certain limitations. First, the proposed network uses the Haar wavelet transform for up- and downsampling. Other potential wavelet transforms include Gaussian, Morlet, Shanno, and other transformation methods, and whether the Haar wavelet transform is the most suitable for MRI prior-based LDPET estimation is not yet known. Second, the proposed network has a significant effect on LDPET images with good overall characteristics but has a poor effect on LDPET images with inconspicuous overall characteristics. The recovery effect of the convolutional neural network on PET images with a dose of $5 \%$ and below needs to be improved; for example, the use of generative adversarial neural networks for image recovery and PET/MRI examinations could reduce the dose even further.

\section{CONCLUSION}

In conclusion, we used a convolutional neural network combining discrete wavelet transform and convolution methods to estimate FDPET images from PSMA LDPET image collected by PET/MRI. After clinical quantitative analysis and objective image index analysis, the deep learning method we proposed was shown to be capable of synthesizing a FDPET image from 50\%-dose PSMA PET images collected by PET/MRI, indicating that the dose can be reduced by $50 \%$.

\section{DATA AVAILABILITY STATEMENT}

Further inquiries can be directed to the corresponding author. Requests to access the datasets should be directed to Zhanli Hu, zl.hu@siat.ac.cn.

\section{ETHICS STATEMENT}

The studies involving human participants were reviewed and approved by (Permit S-152/2020) Department of Nuclear Medicine, Nanjing First Hospital, Nanjing Medical University. The patients/participants provided their written informed consent to participate in this study.

\section{AUTHOR CONTRIBUTIONS}

FD was responsible for programming and revising the manuscript. X-yL and FY performed data acquisition and analyzed the data. HS, JY, QH, YY, DL, XL, GM, HZ and WX made a substantial contribution to the study and helped revise the manuscript critically for important intellectual content. $\mathrm{ZH}$ made substantial contributions to the conception and design of the study. All authors read and approved the final manuscript.

\section{FUNDING}

This work was supported by the National Natural Science Foundation of China (32022042, 81871441), the Shenzhen Excellent Technological Innovation Talent Training Project of 
China (RCJC20200714114436080), the Natural Science Foundation of Guangdong Province in China
(2020A1515010733), Chinese Academy of Sciences Key Laboratory of Health Informatics in China (2011DP173015).

\section{REFERENCES}

1. Sung H, Ferlay J, Siegel RL, Laversanne M, Soerjomataram I, Jemal A, et al. Global Cancer Statistics 2020: GLOBOCAN Estimates of Incidence and Mortality Worldwide for 36 Cancers in 185 Countries. CA: A Cancer J Clin (2021) 71(3):209-49. doi: 10.3322/caac.21660

2. Eiber M, Weirich G, Holzapfel K, Souvatzoglou M, Haller B, Rauscher I, et al. Simultaneous 68ga-PSMA HBED-CC PET/MRI Improves the Localization of Primary Prostate Cancer. Eur Urol (2016) 70(5):829-36. doi: 10.1016/ j.eururo.2015.12.053

3. Hicks RM, Simko JP, Westphalen AC, Nguyen HG, Greene KL, Zhang L, et al. Diagnostic Accuracy of 68Ga-PSMA-11 PET/MRI Compared With Multiparametric MRI in the Detection of Prostate Cancer. Radiology (2018) 289(3):730-7. doi: 10.1148/radiol.2018180788

4. Park SY, Zacharias C, Harrison C, Fan RE, Kunder C, Hatami N, et al. Gallium 68 PSMA-11 PET/MR Imaging in Patients With Intermediate- or High-Risk Prostate Cancer. Radiology (2018) 288(2):495-505. doi: 10.1148/radiol.2018172232

5. Murthy V, Sonni I, Jariwala N, Juarez R, Reiter RE, Raman SS, et al. The Role of PSMA PET/CT and PET/MRI in the Initial Staging of Prostate Cancer. Eur Urol Focus (2021) 7(2):258-66. doi: 10.1016/j.euf.2021.01.016

6. Grubmüller B, Baltzer P, Hartenbach S, D'Andrea D, Helbich TH, Haug AR, et al. PSMA Ligand PET/MRI for Primary Prostate Cancer: Staging Performance and Clinical Impact. Clin Cancer Res (2018) 24(24):6300. doi: 10.1158/1078-0432.CCR-18-0768

7. Kranzbühler B, Nagel H, Becker AS, Müller J, Huellner M, Stolzmann P, et al. Clinical Performance of 68Ga-PSMA-11 PET/MRI for the Detection of Recurrent Prostate Cancer Following Radical Prostatectomy. Eur J Nucl Med Mol Imaging (2018) 45(1):20-30. doi: 10.1007/s00259-017-3850-x

8. Wang R, Shen G, Yang R, Ma X, Tian R. 68ga-PSMA PET/MRI for the Diagnosis of Primary and Biochemically Recurrent Prostate Cancer: A MetaAnalysis. Eur J Radiol (2020) 130:109131. doi: 10.1016/j.ejrad.2020.109131

9. Vandenberghe S, Marsden PK. PET-MRI: A Review of Challenges and Solutions in the Development of Integrated Multimodality Imaging. Phys Med Biol (2015) 60(4):R115. doi: 10.1088/0031-9155/60/4/R115

10. Chen H, Zhang Y, Chen Y, Zhang J, Zhang W, Sun H, et al. LEARN: Learned Experts' Assessment-Based Reconstruction Network for Sparse-Data CT. IEEE Trans Med Imaging (2018) 37(6):1333-47. doi: 10.1109/TMI.2018.2805692

11. Ran M, Xia W, Huang Y, Lu Z, Bao P, Liu Y, et al. Md-Recon-Net: A Parallel Dual-Domain Convolutional Neural Network for Compressed Sensing Mri. IEEE Trans Radiat Plasma Med Sci (2020) 5(1):120-35.

12. Xia W, Lu Z, Huang Y, Liu Y, Chen H, Zhou J, et al. CT Reconstruction With PDF: Parameter-Dependent Framework for Data From Multiple Geometries and Dose Levels. IEEE Trans Med Imaging (2021) 40(11):3065-76. doi: 10.1109/ISBI48211.2021.9433906

13. Xia W, Lu Z, Huang Y, Shi Z, Liu Y, Chen H, et al. MAGIC: Manifold and Graph Integrative Convolutional Network for Low-Dose CT Reconstruction. IEEE Trans Med Imaging (2021) 40(12):3459-72. doi: 10.1109/TMI.2021.3088344

14. Shen C, Xia W, Ye H, Hou M, Chen H, Liu Y, et al. Unsupervised PET Reconstruction From a Bayesian Perspective. arXiv preprint arXiv (2021), 2110.15568.

15. An L, Zhang P, Adeli E, Wang Y, Ma G, Shi F, et al. Multi-Level Canonical Correlation Analysis for Standard-Dose PET Image Estimation. IEEE Trans Image Process (2016) 25(7):3303-15. doi: 10.1109/TIP.2016.2567072

16. Wang Y, Zhang P, An L, Ma G, Kang J, Shi F, et al. Predicting Standard-Dose PET Image From Low-Dose PET and Multimodal MR Images Using Mapping-Based Sparse Representation. Phys Med Biol (2016) 61(2):791812. doi: 10.1088/0031-9155/61/2/791

17. Xiang L, Qiao Y, Nie D, An L, Lin W, Wang Q, et al. Deep Auto-Context Convolutional Neural Networks for Standard-Dose PET Image Estimation From Low-Dose PET/MRI. Neurocomputing (2017) 267:406-16. doi: 10.1016/ j.neucom.2017.06.048

18. Wang Y, Yu B, Wang L, Zu C, Lalush DS, Lin W, et al. 3D Conditional Generative Adversarial Networks for High-Quality PET Image Estimation at Low Dose. NeuroImage (2018) 174:550-62. doi: 10.1016/j.neuroimage.2018.03.045
19. Cui J, Gong K, Guo N, Wu C, Meng X, Kim K, et al. PET Image Denoising Using Unsupervised Deep Learning. Eur J Nucl Med Mol Imaging (2019) 46:2780-9. doi: 10.1007/s00259-019-04468-4

20. Yang L, Xue D, Tonghe W, Kristin H, Tian L, Walter JC, et al. Estimating Standard-Dose PET From Low-Dose PET With Deep Learning. Proc.SPIE (2020) 11313. doi: 10.1117/12.2548461

21. Zhou L, Schaefferkoetter JD, Tham IWK, Huang G, Yan J. Supervised Learning With Cyclegan for Low-Dose FDG PET Image Denoising. Med Image Anal (2020) 65:101770. doi: 10.1016/j.media.2020.101770

22. Borrelli $\mathrm{P}$, Larsson $\mathrm{M}$, Ulén J, Enqvist $\mathrm{O}$, Trägårdh $\mathrm{E}$, Poulsen $\mathrm{MH}$, et al. Artificial Intelligence-Based Detection of Lymph Node Metastases by PET/CT Predicts Prostate Cancer-Specific Survival. Clin Physiol Funct Imaging (2021) 41(1):62-7. doi: 10.1111/cpf.12666

23. Polymeri E, Sadik M, Kaboteh R, Borrelli P, Enqvist O, Ulén J, et al. Deep Learning-Based Quantification of PET/CT Prostate Gland Uptake: Association With Overall Survival. Clin Physiol Funct Imaging (2020) 40 (2):106-13. doi: 10.1111/cpf.12611

24. Han S, Oh JS, Lee JJ. Diagnostic Performance of Deep Learning Models for Detecting Bone Metastasis on Whole-Body Bone Scan in Prostate Cancer. Eur J Nucl Med Mol Imaging (2021). doi: 10.1007/s00259-021-05481-2

25. Kostyszyn D, Fechter T, Bartl N, Grosu AL, Gratzke C, Sigle A, et al. Intraprostatic Tumor Segmentation on PSMA PET Images in Patients With Primary Prostate Cancer With a Convolutional Neural Network. J Nucl Med (2021) 62(6):823. doi: 10.2967/jnumed.120.254623

26. Moazemi S, Essler M, Schultz T, Bundschuh RA, et al. Predicting Treatment Response in Prostate Cancer Patients Based on Multimodal PET/CT for Clinical Decision Support. In: T Cham, Syeda-Mahmood, editors. Multimodal Learning for Clinical Decision Support. Springer International Publishing (2021). p. 22-35.

27. Pozaruk A, Pawar K, Li S, Carey A, Cheng J, Sudarshan V, et al. Augmented Deep Learning Model for Improved Quantitative Accuracy of MR-Based PET Attenuation Correction in PSMA PET-MRI Prostate Imaging. Eur J Nucl Med Mol Imaging (2021) 48(1):9-20. doi: 10.1007/s00259-020-04816-9

28. Liu W, Yan Q, Zhao Y. Densely Self-Guided Wavelet Network for Image Denoising. In: Proceedings of the IEEE/CVF Conference on Computer Vision and Pattern Recognition Workshops (2020). p. 432-3.

29. G R, Lee GR, Wasilewski F, Wohlfahrt K, O'Leary A. PyWavelets: A Python Package for Wavelet Analysis. J Open Source Software (2019) 4:1237. doi: 10.21105/joss.01237

30. Park B, Yu S, Jeong J. Densely Connected Hierarchical Network for Image Denoising. In: Proceedings of the IEEE/CVF Conference on Computer Vision and Pattern Recognition Workshops (2019).

31. Johnson J, Alahi A, Fei-Fei L. Perceptual Losses for Real-Time Style Transfer and Super-Resolution. In: CB Leibe, J Matas, N Sebe, M Welling, editors. Computer Vision - ECCV 2016. Springer International Publishing (2016). p. 694-711.

32. Ouyang J, Chen KT, Gong E, Pauly J, Zaharchuk G. Ultra-Low-Dose PET Reconstruction Using Generative Adversarial Network With Feature Matching and Task-Specific Perceptual Loss. Med Phys (2019) 46(8):355564. doi: 10.1002/mp.13626

33. Chen KT, Gong E, de Carvalho Macruz F, Xu B, Boumis J, Khalighi A, et al. Ultra-Low-Dose 18f-Florbetaben Amyloid PET Imaging Using Deep Learning With Multi-Contrast MRI Inputs. Radiology (2018) 290(3):64956. doi: 10.1148/radiol.2018180940

Conflict of Interest: HS, JY, and QH are employees of the United Imaging Healthcare group.

The remaining authors declare that the research was conducted in the absence of any commercial or financial relationships that could be construed as a potential conflict of interest.

Publisher's Note: All claims expressed in this article are solely those of the authors and do not necessarily represent those of their affiliated organizations, or those of the publisher, the editors and the reviewers. Any product that may be evaluated in 
this article, or claim that may be made by its manufacturer, is not guaranteed or endorsed by the publisher.

Copyright $\odot 2022$ Deng, Li, Yang, Sun, Yuan, He, Xu, Yang, Liang, Liu, Mok, Zheng and $H u$. This is an open-access article distributed under the terms of the Creative
Commons Attribution License (CC BY). The use, distribution or reproduction in other forums is permitted, provided the original author(s) and the copyright owner(s) are credited and that the original publication in this journal is cited, in accordance with accepted academic practice. No use, distribution or reproduction is permitted which does not comply with these terms. 